\title{
Full jet tomography of high-energy nuclear collisions
}

\author{
Ben-Wei Zhang \\ Institute of Particle Physics, Central China Normal University, Wuhan 430079, China \\ Key Laboratory of Quark \& Lepton Physics (Central China Normal University), Ministry of Education, China
}

\begin{abstract}
Parton energy loss in the hot QCD medium will manifest itself not only in leading hadron spectra but also in reconstructed jet productions in high-energy nucleus-nucleus collisions. In this paper we report on recent theoretical efforts in studying full jet observables in relativistic heavy-ion collisions by discussing the modifications of jet shapes, inclusive jet cross section and the vector boson accompanied jet production in the presence of the QGP-induced jet quenching.
\end{abstract}

Keywords: jet quenching, reconstructed jet, perturbative QCD

\section{Introduction}

In high-energy nucleus-nucleus collisions a deconfined matter, the quark-gluon plasma (QGP) is to be formed and when an energetic parton produced by the initial hard scattering propagates in this hot and dense QCD medium, it will interact with other partons in the hot nuclear medium and be quenched substantially[1, 2, 3, 4, 5]. This parton energy loss mechanism, or jet quenching, has been proposed to be an excellent probe of the QGP and predicted the suppression of single hardon productions and the disappearance of away-side dihadron correlations in heavy-ion collisions (HIC), which later have been confirmed by the experimental measurements at RHIC and given compelling evidence of the existence of a new kind of matter in high-energy nuclear collisions at RHIC [6]. Not surprisingly, the finding of jet quenching in heavy-ion collisions has been regarded as one of the most important discoveries made at RHIC and initiated many theoretical explorations and very precise experimental measurements [7].

So far the dominant experimental measurements of jet quenching is about the production of one or two hadrons with a large transverse momentum, which are only the leading fragments of a jet. The parton energy loss mechanism will not only manifest itself in leading particle productions, but more interestingly in full jet observables. Recently in heavy-ion community a large amount of effort has been invested in measuring reconstructed jets in high-energy nuclear collisions for the first time [8, 9, 10], and theories to address the novel features of full jet tomography in relativistic heavy-ion collisions have emerged. In this article we review our theoretical studies on jet observables of high-energy nucleus-nucleus collisions in perturbative Quantum Chromodynamics (pQCD) by focusing on the intra-jet energy flow, the inclusive jet cross section and $Z^{0} / \gamma^{*}$-tagged jet production [11, 12, 13] as well as possible non-perturbative effects in jet productions. 

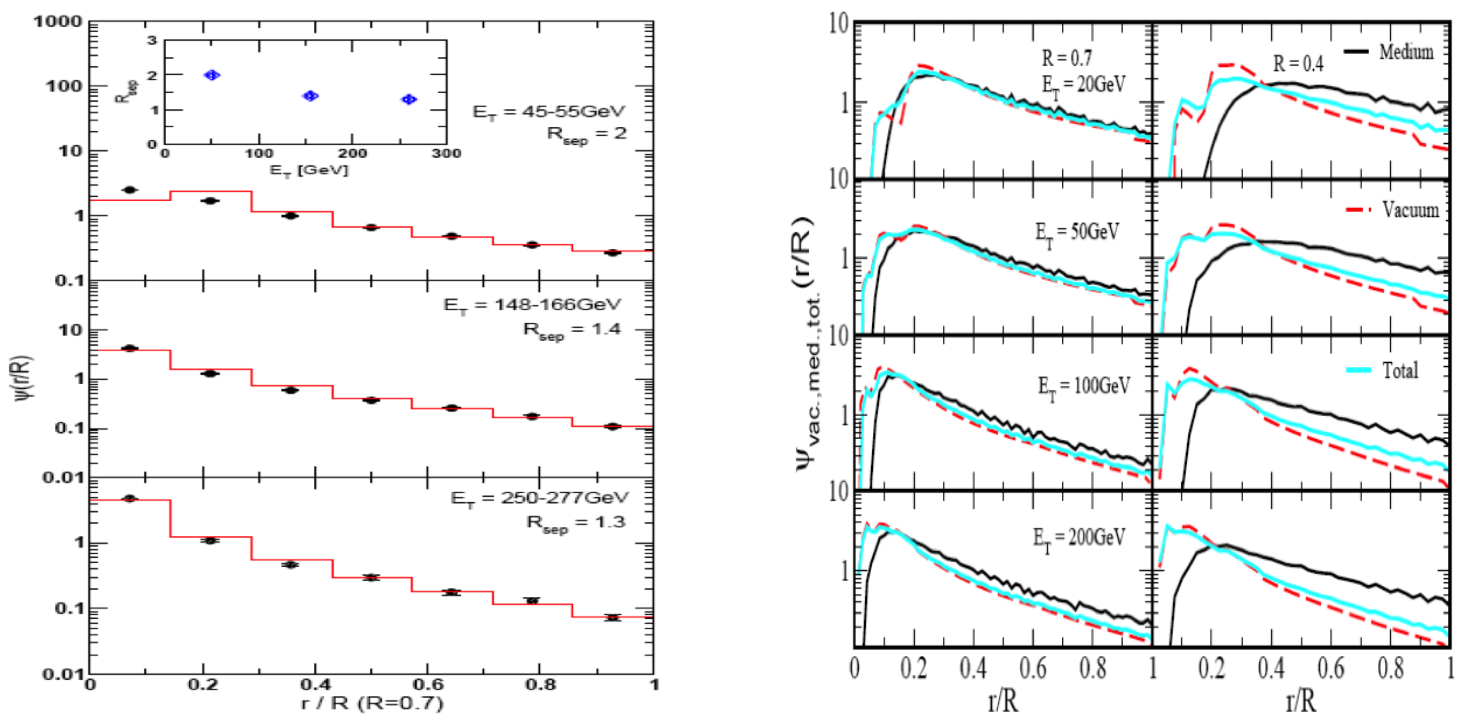

Figure 1: Comparison of numerical results from our theoretical calculation to experimental data on differential jet shapes at $\sqrt{s}=1960 \mathrm{GeV}$ by $\mathrm{CDF}$ II [18] (left panel). Numerical simulation of jet shapes in vacuum, medium-induced jet shapes, and total jet shapes in medium for $\mathrm{Pb}+\mathrm{Pb}$ with $\sqrt{s_{N N}}=5.5 \mathrm{TeV}$ at LHC (right panel).

\section{Jet shapes in HIC}

One of the most common obesevables of resolving the internal jet structure is the jet shape, which describes the energy distribution in a jet as [14]:

$$
\Psi_{\text {int }}(r ; R)=\frac{\sum_{i}\left(E_{T}\right)_{i} \Theta\left(r-R_{i, \text { jet }}\right)}{\sum_{i}\left(E_{T}\right)_{i} \Theta\left(R-R_{i, \text { jet }}\right)} .
$$

Here $r, R$ are Lorentz-invariant opening angles, $R_{i \text {,jet }}=\sqrt{\left(y_{i}-y_{\text {jet }}\right)^{2}+\left(\phi_{i}-\phi_{\text {jet }}\right)^{2}}$ gives the distance between a parton $i$ and the jet in the space of rapidity $y$ and azimuthal $\phi$.

In leading order we can derive the differential jet shape as [11]

$$
\psi(r ; R)=\frac{d \Psi_{\mathrm{int}}(r ; R)}{d r}=\sum_{b} \frac{\alpha_{s}}{2 \pi} \frac{2}{r} \int_{z_{\min }}^{1-Z} d z z P_{a \rightarrow b c}(z),
$$

where

$$
\begin{aligned}
& Z=\max \left\{z_{\min }, \frac{r}{r+R}\right\} \text { if } r<\left(R_{\text {sep }}-1\right) R \\
& Z=\max \left\{z_{\min }, \frac{r}{R_{\text {sep }} R}\right\} \text { if } r>\left(R_{\text {sep }}-1\right) R
\end{aligned}
$$

Here $1 \leq R_{\text {sep }} \leq 2$ is introduced to take into account features of experimental cone algorithms, employed to improve infrared safety. In Eq. (2) $r=(1-z) \rho$ is related to the opening angle $\rho$ between the final-state partons, $P_{a \rightarrow b c}(z)$ is the splitting kernel in DGLAP evolution equation, $z$ is the momentum fraction of the radiated parton relative to the parent parton, $z_{\text {min }}=E_{T}^{\min } / E_{T}$ with $E_{T}$ representing the transverse momentum of a jet, and $E_{T}^{\text {min }}$ the minimum transverse momentum of a parton in this jet .

Carrying out the integration of $z$ we get the jet shapes at LO. For instance, the jet shape for a quark gives:

$$
\psi_{q}(r)=\frac{C_{F} \alpha_{s}}{2 \pi} \frac{2}{r}\left(2 \log \frac{1-z_{\min }}{Z}-\frac{3}{2}\left[(1-Z)^{2}-z_{\text {min }}^{2}\right]\right),
$$




\begin{tabular}{|c|l|l|l|}
\hline $\mathrm{R}=0.4$ & Vacuum & $\begin{array}{c}\text { Complete } \\
\text { E-loss }\end{array}$ & $\begin{array}{c}\text { Realistic } \\
\text { Case }\end{array}$ \\
\hline$<r / R>, E_{T}=20 \mathrm{GeV}$ & 0.41 & 0.55 & 0.45 \\
\hline$<r / R>, E_{T}=50 \mathrm{GeV}$ & 0.35 & 0.48 & 0.38 \\
\hline$<r / R>, E_{T}=100 \mathrm{GeV}$ & 0.28 & 0.44 & 0.32 \\
\hline$<r / R>, E_{T}=200 \mathrm{GeV}$ & 0.25 & 0.40 & 0.28 \\
\hline \hline $\mathrm{R}=0.7$ & Vacuum & $\begin{array}{c}\text { Complete } \\
\text { E-loss }\end{array}$ & Realistic \\
\hline$<r / R>, E_{T}=20 \mathrm{GeV}$ & 0.41 & 0.44 & 0.42 \\
\hline$<r / R>, E_{T}=50 \mathrm{GeV}$ & 0.33 & 0.39 & 0.37 \\
\hline$<r / R>, E_{T}=100 \mathrm{GeV}$ & 0.27 & 0.34 & 0.29 \\
\hline$<r / R>, E_{T}=200 \mathrm{GeV}$ & 0.24 & 0.31 & 0.26 \\
\hline
\end{tabular}

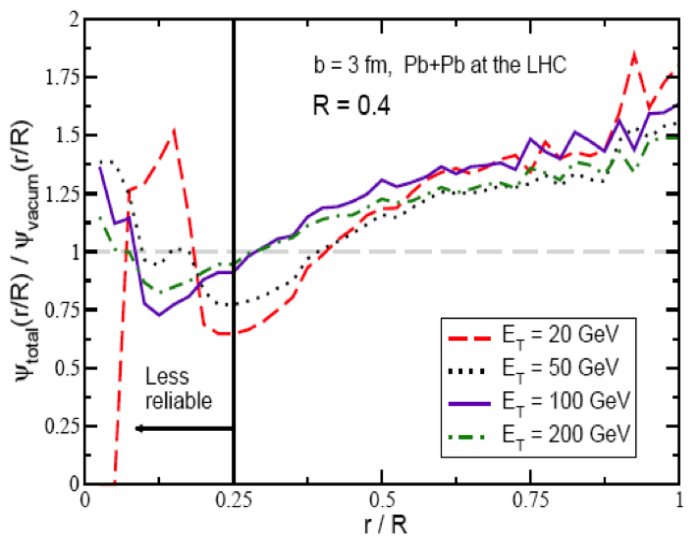

Figure 2: The mean relative jet radii $\langle r / R\rangle$ in the vacuum, with complete energy loss, and in the QGP medium for $\sqrt{s}=5.5 \mathrm{TeV}$ central $\mathrm{Pb}+\mathrm{Pb}$ collisions at the LHC (left panel); The ratios of total jet shape in heavy-ion collisions at LHC to the jet shape in the vacuum (right panel).

Eq. (3) is collinear divergent when $r \rightarrow 0$. To get rid of this divergence, we need to make Sudakov resummation. Also we could improve the prediction power of our analysis by estimating the non-perturbative effect and include contributions of the initial-state radiation and corresponding Sudakov resummation [14]. Taking into account all these contributions, we do numerical simulation and show the comparison of the theoretical results for jet shapes in $p+\bar{p}$ collisions with $\sqrt{s_{N N}}=1960 \mathrm{GeV}$ at Tevatron with CDF II data in Fig. 1. It could be seen that the theoretical model describes CDF II data very well, especially jet shapes away from $r=0$ [11].

In a hot and dense QCD medium, a propagating fast parton may lose a large amount of energy by mediuminduced gluon radiation, and some lost energy carried away by radiated gluon may be recaptured by the jet and thus gives additional contributions to jet shapes. In our studies, we calculate the radiated gluon angular distribution in medium within the GLV formalism [4] and compute the medium-induced jet shapes as well as the in-medium jet cross sections (see Sec 3 and Sec 4) using full numerical evaluation of the medium-induced contribution to the parton showers. In the model energetic inclusive jet production and tagged jet production are rare processes that follow binary collision scaling $\sim d^{2} N_{\text {bin }} / d^{2} \mathbf{x}$ with $d^{2} \mathbf{x}$ representing the area in the transverse plane; in contrast, the medium is distributed according to the number of participants density $\sim d^{2} N_{\text {part }} / d^{2} \mathbf{x}$ and the longitudinal Bjorken expansion of the QGP incorporated.

The total jet shapes in HIC then is given by[11]:

$$
\psi_{\text {tot. }}\left(\frac{r}{R}\right) \propto \int_{\epsilon=0}^{1} d \epsilon \sum_{q, g} \frac{P_{q, g}(\epsilon, E)}{\left(1-\left(1-f_{q, g}\right) \cdot \epsilon\right)^{3}}\left[(1-\epsilon) \psi_{\text {vac. }}^{q, g}\left(\frac{r}{R} ; E^{\prime}\right)+f_{q, g} \cdot \epsilon \psi_{\text {med. }}^{q, g}\left(\frac{r}{R} ; E^{\prime}\right)\right],
$$

where $\left(1-f_{q, g}\right) \cdot \epsilon$ gives the fraction of the energy of the parent parton falling outside of jet area with a radius $R$. In the right panel of Fig. 1 1 we illustrate jet shapes in vacuum $\psi_{\text {vac. }}(r / R)$, medium-induced jet shapes $\psi_{\text {med. }}(r / R)$, and total jet shapes in HIC $\psi_{\text {tot. }}(r / R)$ in $\mathrm{Pb}+\mathrm{Pb}$ collisions with $\sqrt{s_{N N}}=5.5 \mathrm{TeV}$. It is observed that though medium-induced jet shapes are quite distinct from jet shapes in vacuum, the difference between jet shapes in $\mathrm{p}+\mathrm{p}$ collisions and that in $\mathrm{Pb}+\mathrm{Pb}$ is not very large. The underlining reason for this surprising result is that although medium-induced gluon radiation produces a broader $\psi_{\text {med. }}(r / R)$, this effect is offset by the fact that jets lose a finite amount of their energy, which will shift jet shapes in vacuum to higher $E_{T}$ with steeper profile. The left panel of Fig. 2 shows the mean relative jet radii $\langle r / R\rangle$ in the vacuum and in the QGP medium created at the LHC for two different cone selections $R=0.4$ and $R=0.7$. We can find that the broadening of radii $\langle r / R\rangle$ of jet shapes in HIC is modest, which implies only a fraction of energy of jets lost in the hot medium and the QGP is rather 'gray' instead of 'black' [11]. The deviation of $\psi_{\text {tot. }}(r / R)$ from $\psi_{\text {vac. }}(r / R)$ is pronounced in the tail of jet shapes for small cone size $R=0.4$, as illustrated in the right panel of Fig. 2 . 

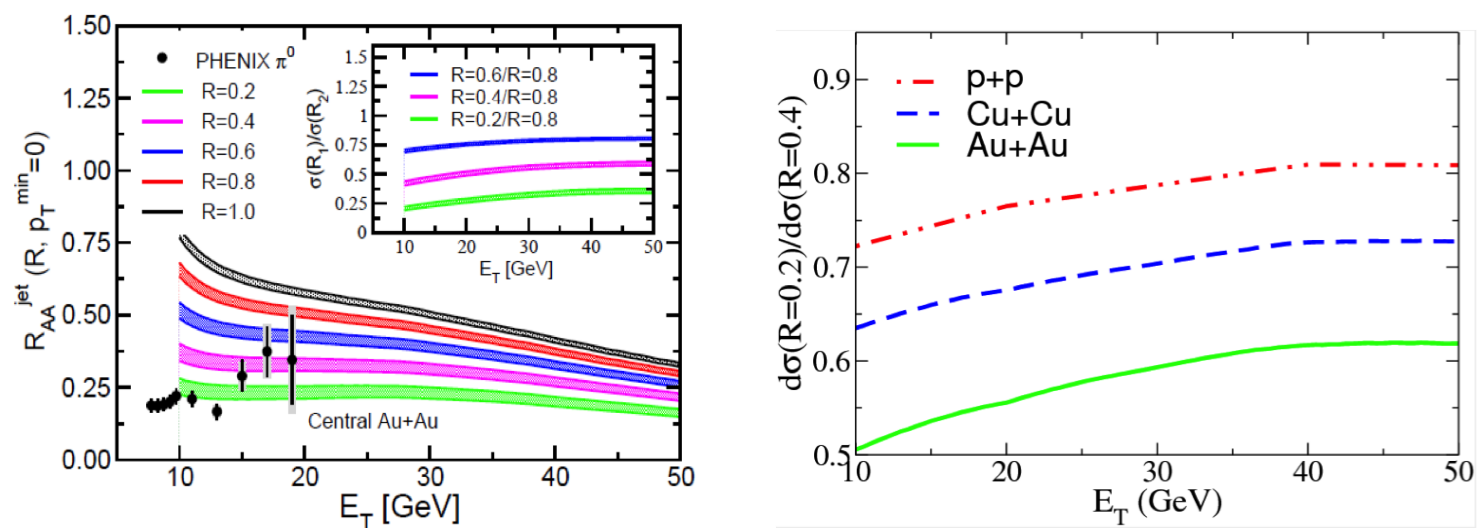

Figure 3: Transverse energy dependent nuclear modification factor $R_{A A}^{\text {jet }}$ for different cone radii $R$ at NLO in $b=3 \mathrm{fm} \mathrm{Au+Au} \mathrm{collisions} \mathrm{at}$ $\sqrt{s_{N N}}=200 \mathrm{GeV}$ (left panel). The ratio of jet yields at $R=0.2$ and $R=0.4 \mathrm{in} \mathrm{p}+\mathrm{p}$, central $\mathrm{Cu}+\mathrm{Cu}$ and $\mathrm{Central} \mathrm{Au}+\mathrm{Au}$ collisions with $\sqrt{s_{N N}}=200 \mathrm{GeV}$ at RHIC (right panel).

\section{Inclusive jet cross sections in HIC}

Parton energy loss in the QGP not only changes the energy distribution in a jet, but also alters the inclusive jet spectrum in high-energy nucleus-nucleus reactions. A first study of inclusive jet productions in HIC was carried out in the leading order (LO) [11], and recently to take full advantage of jet physics in reactions with ultra-relativistic nuclei a next-to-leading (NLO) calculations at $O\left(\alpha_{s}^{3}\right)$ have been made [12].

In hadron-hadron collisions the inclusive jet cross section at NLO can be given as [15]:

$$
\frac{d \sigma^{\mathrm{jet}}}{d E_{T} d y}=\frac{1}{2 !} \int d\left\{E_{T}, y, \phi\right\}_{2} \frac{d \sigma[2 \rightarrow 2]}{d\left\{E_{T}, y, \phi\right\}_{2}} S_{2}\left(\left\{E_{T}, y, \phi\right\}_{2}\right)+\frac{1}{3 !} \int d\left\{E_{T}, y, \phi\right\}_{3} \frac{d \sigma[2 \rightarrow 3]}{d\left\{E_{T}, y, \phi\right\}_{3}} S_{3}\left(\left\{E_{T}, y, \phi\right\}_{3}\right),
$$

where $E_{T i}, y_{i}, \phi_{i}$ are the transverse energy, rapidity, and azimuthal angle of the $\mathrm{i}$-th particle $(i=1,2,3)$, respectively, and $\sigma[2 \rightarrow 2], \sigma[2 \rightarrow 3]$ stand for the partonic cross sections. In Eq. (5) $S_{2}, S_{3}$ give the space constraints and contain the information of the jet-finding algorithm. At leading order, a jet is equivalent to a parton and we always have $S_{2}=\sum_{i=1}^{2} S(i)=\sum_{i=1}^{2} \delta\left(E_{T_{i}}-E_{T}\right) \delta\left(y_{i}-y\right)$. At NLO due to higher order corrections $S_{3}$ has two contributions: one coming from the possibility that each jet still contains one parton; another resulting from the chance that a jet contains two partons imposed by the jet finding algorithm [21]. Numerical simulation shows that the inclusive jet spectrum in $\mathrm{p}+\mathrm{p}$ collisions with $\sqrt{s_{N N}}=200 \mathrm{GeV}$ at RHIC is described very well by a NLO code of jet production in hadronic collisions [15], which will later be extended to study inclusive jet productions at NLO in HIC.

In the same spirit of Eq. (4) for jet shapes in HIC, we derive the related medium-modified jet cross section as follows [12]:

$$
\frac{1}{\left\langle T_{A A}\right\rangle \sigma_{A A}^{\mathrm{geo}}} \frac{d \sigma^{A A}(R)}{d^{2} E_{T} d y}=\int_{\epsilon=0}^{1} d \epsilon \sum_{q, g} P_{q, g}(\epsilon, E) \frac{1}{\left(1-\left(1-f_{q, g}\right) \cdot \epsilon\right)^{2}} \frac{d \sigma_{q, g}^{\mathrm{CNM}, \mathrm{NLO}}(R)}{d^{2} E_{T}^{\prime} d y} .
$$

Here $T_{A A}$ is the nuclear overlap function, and $\sigma_{A A}^{\text {geo }}$ the geometrical $\mathrm{A}+\mathrm{A}$ cross section [16]. The measured cross section is then a probabilistic superposition of the cross sections of protojets of initially larger energy $E_{T}^{\prime}=E_{T} /\left(1-\left(1-f_{q, g}\right) \cdot \epsilon\right)$.

Now we are ready to compute the nuclear modification factor of jet cross section $R_{A A}^{\text {jet }}$ defined as the ratio of jet cross section in HIC to that in $\mathrm{p}+\mathrm{p}$ rescaled by the number of binary collisions. The numerical results for the nuclear modification factor of inclusive jets $R_{A A}^{\mathrm{jet}}$ in central $\mathrm{Au}+\mathrm{Au}$ with $\sqrt{s_{N N}}=200 \mathrm{GeV}$ at RHIC are presented in the left panel of Fig. 3] [12], where the band of theoretical calculations shows a calculation for a $\sim 20 \%$ increase in the rate of parton energy loss relative to our default simulation. A continuous variation of $R_{A A}^{\text {jet }}$ with the jet radius $R$ is found, which demonstrates a unique feature of jet production as compared to the single curve of $R_{A A}$ for pion production in HIC. In the calculations several cold nuclear matter (CNM) effects such as nuclear shadowing, Cronin effect and 

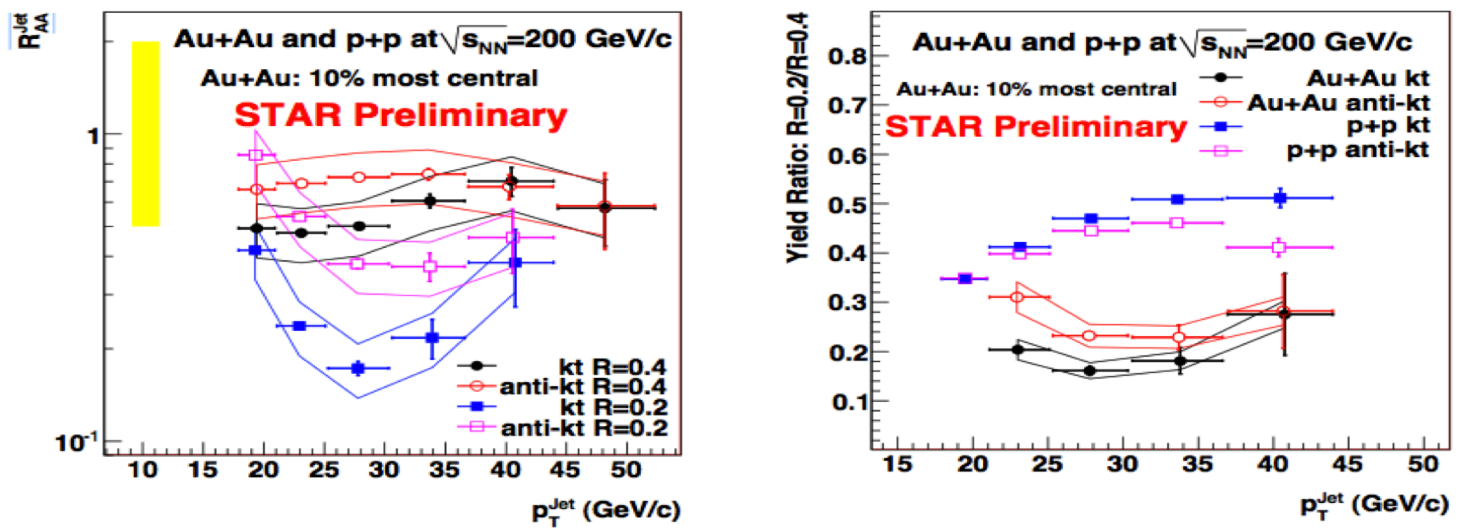

Figure 4: Experimental measurements of full jet production in HIC with $\sqrt{s_{N N}}=200 \mathrm{GeV}$ at RHIC [8] 10]: the nuclear modification factor of jet production in $\mathrm{Au}+\mathrm{Au}$ collisions at different jet radii (left panel); ratios of jet cross sections with $R=0.2$ to that with $R=0.4 \mathrm{in} \mathrm{p}+\mathrm{p}$ and $\mathrm{Au}+\mathrm{Au}$ collisions (right panel).

EMC effect have been taken into account [19, 20]. We observe that for $R \leq 0.2$ the quenching of jets approximates the already observed suppression in the production rate of inclusive high- $p_{T}$ particles. In our theoretical calculation CNM effects contribute significantly to the observed attenuation at large $E_{T}$. We could suppress the effects of CNM by taking the ratios of jet cross sections at different cone radii, which are plotted in the inserts of the left panel of Fig. 3 .

To see the distinction of jet productions in different systems more clearly we plot the ratio $\frac{d \sigma^{A A}(R=0.2)}{d E_{T} d y} / \frac{d \sigma^{A A}(R=0.4)}{d E_{T} d y}$ for $\mathrm{p}+\mathrm{p}$ collisions, central $\mathrm{Cu}+\mathrm{Cu}$ and central $\mathrm{Au}+\mathrm{Au}$ collisions with $\sqrt{s_{N N}}=200 \mathrm{GeV}$ at RHC in the right panel of Fig. 3. It shows that the ratio decreases as the system becomes larger, and the ratio in $\mathrm{Au}+\mathrm{Au}$ at RHIC is smaller than those in $\mathrm{p}+\mathrm{p}$ and $\mathrm{Cu}+\mathrm{Cu}$ collision at the same colliding energy, which implies in HIC more contributions of jet yields come from large angular radiation as compared to $\mathrm{p}+\mathrm{p}$ collisions.

Recently PHENIX and STAR have measured reconstructed jets in high-energy nucleus-nucleus collisions at RHIC and some preliminary results [8, 10] are illustrated in Fig. 4] From the left panel of Fig. 4] one can see that measured $R_{A A}^{\mathrm{jet}}$ changes with the jet radius and at smaller radius the nuclear modification factor of jets is smaller, which agrees with our theoretical prediction [11, 12] shown in the left panel of Fig. 3 Moreover the experimental measurements also demonstrate the ratio of jet yields at different radii in $\mathrm{Au}+\mathrm{Au}$ is smaller than the one in $\mathrm{p}+\mathrm{p}$ (see the right panel of Fig. 4) which confirms the interesting feature shown the right panel of Fig. 3 with our theoretical study. We notice that though our theoretical predictions of inclusive jets describe very well the overall trends of the jet measurements at RHIC, some deviations exist if we compare the results in Fig. 3 with the data in Fig. 4. To confront the theoretical studies with the experimental data on a more solid base, we should include the correction from non-perturbative effects of jet productions [21, 22, 23] in our theoretical investigation (please see Sec 5 for more discussions), and precise experimental measurements of jets in HIC with large statistics will also be needed.

\section{Tagged jet productions in HIC}

A natural way to extend the study of the inclusive jet production in high-energy nucleus-nucleus reactions is to consider tagged jet productions in HIC, especially the jet production accompanied with a vector boson $\left(\gamma\right.$ or $\left.Z^{0}\right)$ in nuclear collisions. In the leading order, the vector boson and the accompanied jet are produced back-to-back in the center-of-mass frame and the transverse momentum of the vector boson $E_{T}^{\mathrm{VB}}$ is the same as that of the tagged jet $E_{T}^{\text {jet }}$ before they travel in the QGP. Because the vector boson doesn't interact with the medium strongly its transverse momentum of the vector boson is intact. By measuring $E_{T}^{\mathrm{VB}}$ and $E_{T}^{\mathrm{jet}}$ in the final stage of heavy-ion collisions we may know exactly the energy loss of the jet by assessing $E_{T}^{\mathrm{VB}}-E_{T}^{\mathrm{jet}}$. Therefore the vector boson + jet production has been considered as an optimal channel to pinpoint the parton energy loss in the QGP and reveal the detailed information of 

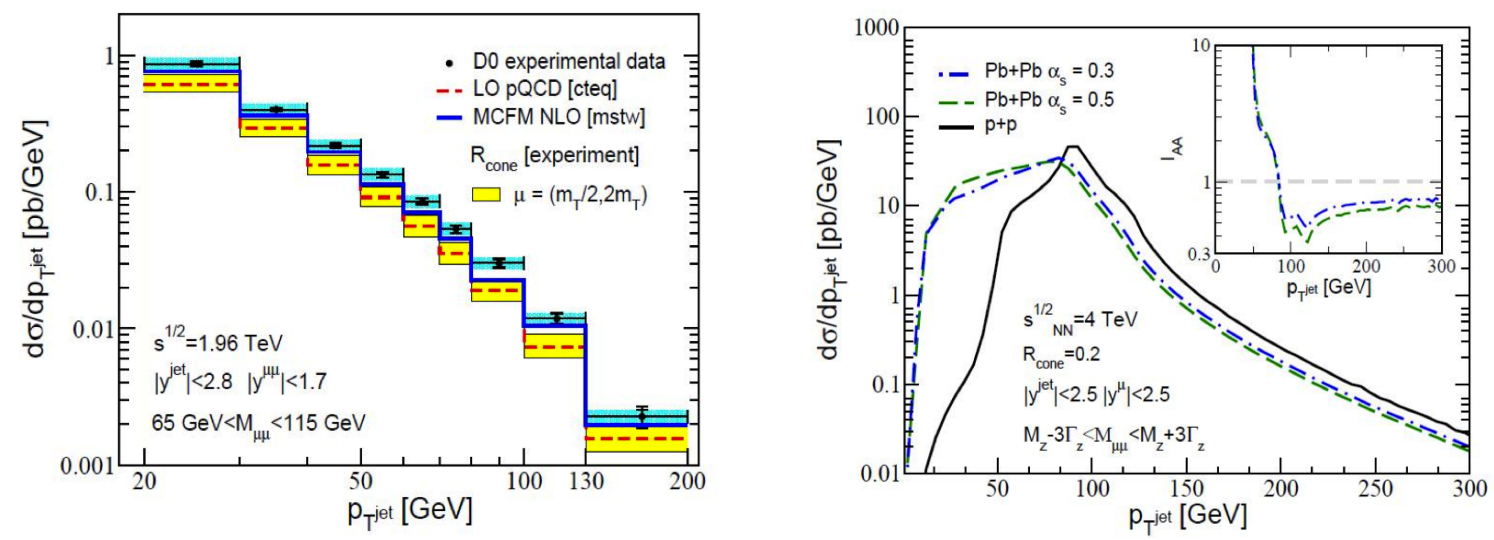

Figure 5: Comparison of the theoretical calculation of $Z^{0} / \gamma^{*}$ tagged jet production in LO and NLO with MCFM to experimental results from the Fermi Lab Tevatron Collider [26] for the cross section of jets associated with $Z^{0} / \gamma^{*} \rightarrow \mu^{+}+\mu^{-}$in $p+\bar{p}$ collisions at $\sqrt{s}=1.96$ TeV (left panel). The NLO $p_{T}$-differential cross section per nucleon pair for jets tagged with $Z / \gamma^{*} \rightarrow \mu^{+}+\mu^{-}$in $\mathrm{p}+\mathrm{p}$ and central $\mathrm{Pb}+\mathrm{Pb}$ reactions when $92.5 \mathrm{GeV}<p_{T}^{\text {jet }}<112.5 \mathrm{GeV}$ (right panel).

the hot and dense QCD medium formed in relativistic HIC. However, higher order corrections will break the momenta balance between tagged jet and the vector boson due to additional radiation. Here we will review our recent study of $Z^{0} / \gamma^{*}+$ jet production in $\mathrm{Pb}+\mathrm{Pb}$ reactions at $\mathrm{NLO}$ and for a more detailed discussion we refer to [13, 24].

For the baseline evaluation of tagged jet cross sections at NLO in hadron-hadron reactions we use the Monte Carlo for FeMtobarn (MCFM) numerical code [25]. The confrontation of theoretical computation of tagged jet at MCFM NLO against the experiment measurement for $p+\bar{p}$ collisions at Tevatron in the left panel of Fig. 5 attests to the validity of pQCD calculations. In heavy-ion collisions the medium-induced parton energy loss will modify the tagged jet spectrum and the quenched jet cross section is given as follows:

$$
\frac{d \sigma^{A A}}{d^{2} \mathbf{p}_{(Z)} d^{2} \mathbf{p}_{Q}}=\sum_{q, g} \int d \epsilon P_{q, g}(\epsilon) \frac{1}{\left[1-\left(1-f_{q, g}\left(\omega_{\min }, R\right)\right) \epsilon\right]^{2}} \frac{d \sigma^{q, g}}{d^{2} \mathbf{p}_{(Z)} d^{2} \mathbf{p}_{(\mathrm{jet})}}\left(\frac{\mathbf{p}_{Q}}{\left.\left[1-\left(1-f_{q, g}\right) \epsilon\right)\right]}\right),
$$

with $\mathbf{p}_{Q}=\mathbf{p}_{\text {(jet) }}\left(1-\left(1-f_{q, g}\right) \epsilon\right)$. Eq. (7) implies that the observed tagged jet cross section in $\mathrm{A}+\mathrm{A}$ reactions is a probabilistic superposition of cross sections for jets of higher initial transverse energy. This excess energy is then redistributed outside of the jet due to strong final-state interactions. Here $d \sigma^{q, g} / d^{2} \mathbf{p}_{(Z)} d^{2} \mathbf{p}_{(\mathrm{jet})}$ are the differential cross sections for away-side quark and gluon jets, respectively.

In some experiments the $Z^{0} / \gamma^{*}+$ jet final-state channel is measured without placing restrictions on the momentum of the vector boson [26]. In this case, we can integrate over $\mathbf{p}_{(Z)}$ in Eq. (7) and derive:

$$
\frac{d \sigma^{A A}}{d^{2} \mathbf{p}_{Q}}=\sum_{q, g} \int d \epsilon P_{q, g}(\epsilon) \frac{1}{\left[1-\left(1-f_{q, g}\left(\omega_{\min }, R\right)\right) \epsilon\right]^{2}} \frac{d \sigma^{q, g}}{d^{2} \mathbf{p}_{(\mathrm{jet})}}\left(\frac{\mathbf{p}_{Q}}{\left.\left[1-\left(1-f_{q, g}\right) \epsilon\right)\right]}\right) .
$$

We notice that the integrated tagged jet cross section $d \sigma^{A A} / d^{2} \mathbf{p}_{Q}$ usually unravels the same physics as the suppression of the cross section in inclusive jet production discussed in Sec. 3 .

To demonstrate nuclear effects for tagged jet cross sections in HIC we define $I_{A A}^{\text {jet }}$ for tagged jet as:

$$
I_{A A}^{\mathrm{jet}}\left(R, \omega_{\mathrm{min}}\right)=\frac{1}{\left\langle T_{A A}\right\rangle \sigma_{A A}^{\mathrm{geo}}} \frac{d \sigma_{A A}}{d p_{T(Z)} d p_{T(Q)}} \mid \frac{d \sigma_{p p}}{d p_{T(Z)} d p_{T(\mathrm{jet})}} .
$$

While at LO $I_{A A}^{\text {jet }}$ is limited to the $Z^{0} / \gamma^{*}$ trigger momentum range, at NLO it will show rich features as we will discuss below.

A numerical simulation of $Z^{0} / \gamma^{*}$ tagged jet productions in $\mathrm{p}+\mathrm{p}$ and $\mathrm{Pb}+\mathrm{Pb}$ with $\sqrt{s_{N N}}=4 \mathrm{TeV}$ for a cone size $R=0.2$ is provided in the right panel of Fig. 5. It is found because part of the jet energy is redistributed outside 

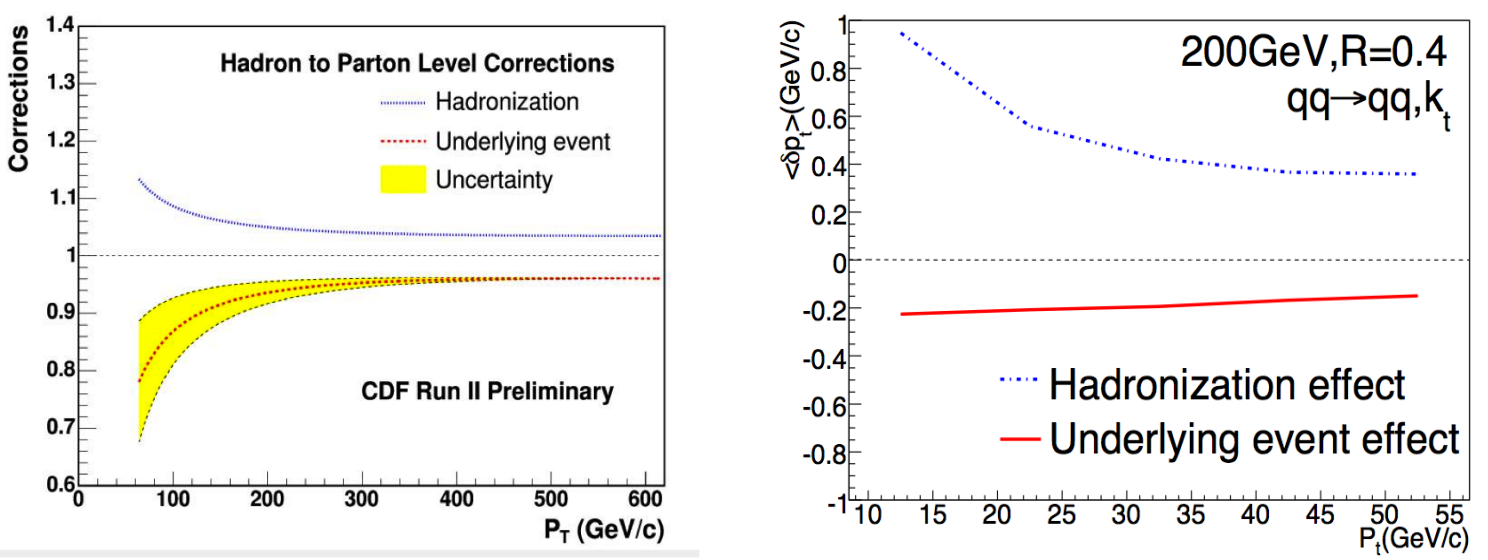

Figure 6: Magnitude of the hadronization and underlying event effects used to correct the inclusive jet cross section measured by CDF with jet radius $R=0.7$ [21] (left panel). Modification of $p_{T}$ of jets due to non-perturbative effects in $p+p$ collisions at RHIC with Pythia8.142 [27] (right panel). Here for all plots the corrections are taken from hadron to parton level.

of the jet cone the $p_{T}$ spectrum of tagged jet production in HIC is downshifted toward smaller transverse momenta. More interestingly one can observe a sharp transition of $I_{A A}^{\text {jet }}$ from tagged jet enhancement below $p_{T(Z)}$ to tagged jet suppression above $p_{T(Z)}$. This striking feature provides a unique prediction of jet quenching for tagged jets and will be experimental evidence for strong final-state interactions and parton energy loss in the QGP. Please note that while the same energy redistribution occurs for inclusive jet production discussed in Sec. 3 , the monotonically falling spectrum prevents the observation of such inclusive jet enhancement.

\section{Non-perturbative effects in jet productions}

In perturbative QCD calculations of inclusive jet yield and tagged jet production, a jet is the combination of a few partons defined by jet algorithms and what we calculate is the parton-jet. However, in experiments what we measured is the hadron-jet since the final-state particles are hadrons. For jet production at high transverse momentum in a hard process with a large momentum transfer, the difference between parton-jets and hadron-jets may be small due to local parton-hadron duality (LPHD) [15, 21]. Nevertheless, to make a more reliable comparison between QCD theory of jet productions and the jet measurements, the theoretical result of jet cross sections with pQCD should be corrected to hadron level, or on the contrary the experimental data should be corrected to parton level [21, 27, 28].

To include these corrections two important non-perturbative effects should be taken into account: the hadronization effect which results in the energy loss of a jet because when partons in a parton-jet hadronize, some hadrons may fall outside of the jet ( a "splashing-out" effect); the underlying event effects which gives the energy gain of a jet due to interaction of beam-beam remnants not associated with the hard scattering ( a "splash-in" effect ). The good news is that the hadronization effect and the underlying event effect go in opposite direction and their contributions will be partially cancelled.

The left panel of Fig. 6 gives the magnitude of non-perturbative effects used to correct the $p_{T}$ spectra with a cone size $R=0.7$ for $p+\bar{p}$ collisions with $\sqrt{s_{N N}}=1960 \mathrm{GeV}$ by CDF at Tevatron. We find that when $p_{T}>200 \mathrm{GeV}$ the fractional corrections due to non-perturbative effects are within few percent. However, this observation holds true only for this special case and corrections from the non-perturbative effects may vary with the colliding energy $\sqrt{s_{N N}}$, jet transverse momenta $p_{T}$ and the jet radius $R$ [23, 27]. At RHIC with colliding energy $200 \mathrm{GeV}$ the measured jet does not have a very large $p_{T}$, and with small jet radii $(R=0.2,0.4$ for measurements in Fig. 4) the contributions from non-perturbative effects may be considerable. In the right panel of Fig. 6 we show a numerical simulation of the jet $p_{T}$ shifting [27] to inclusive jet production due to the hadronization effect and the underlying event effects in $p+p$ collisions at RHIC with Pythia8.142 [29]. It could be seen that when $p_{T} \sim 12 \mathrm{GeV}$ the relative shifting $\delta p_{t} / p_{T}$ due to hadronization could be about $8 \%$, and its effect will be significantly amplified by the steeply falling $p_{T}$ spectrum of 
jets when we studying the jet cross section. Thus it is important to take into account corrections of non-perturbative effects to jet productions in HIC at RHIC and see how jet spectra will be modified by these corrections [27].

Acknowledgments: The work is finished in collaboration with I. Vitev, S. Wicks and B. Neufeld. I thank X. N. Wang, J. W. Qiu and G. Soyez for illuminating discussions. This research is supported by the Ministry of Education of China with the Program NCET-09-0411, by National Natural Science Foundation of China with Project No. 11075062, and CCNU with Project No. CCNU09A02001.

\section{References}

[1] X. -N. Wang, M. Gyulassy, Phys. Rev. Lett. 68, 1480-1483 (1992).

[2] M. Gyulassy, I. Vitev, X. N. Wang and B. W. Zhang, arXiv:nucl-th/0302077

[3] R. Baier, Y. L. Dokshitzer, A. H. Mueller, S. Peigne and D. Schiff, Nucl. Phys. B 484, 265 (1997); B. G. Zakharov, JETP Lett. 73, 49 (2001) [Pisma Zh. Eksp. Teor. Fiz. 73, 55 (2001)]; N. Armesto, C. A. Salgado and U. A. Wiedemann, Phys. Rev. D 69, 114003 (2004).

[4] M. Gyulassy, P. Levai and I. Vitev, Phys. Rev. Lett. 85, 5535 (2000); I. Vitev, Phys. Rev. C 75, 064906 (2007).

[5] X. N. Wang and X. F. Guo, Nucl. Phys. A 696, 788 (2001); B. W. Zhang and X. N. Wang, Nucl. Phys. A 720, 429 (2003); B. W. Zhang, E. k. Wang and X. N. Wang, Nucl. Phys. A 757, 493 (2005).

[6] RHIC WHITE REPORT, Nucl. Phys. A757, 1-283 (2005).

[7] D. d'Enterria, arXiv:0902.2011 [nucl-ex]].

[8] S. Salur, Nucl. Phys. A 830, 139C (2009); M. Ploskon, Nucl. Phys. A 830, 255C (2009); Y. -S. Lai [ PHENIX Collaboration ], Nucl. Phys. A830, 251C-254C (2009).

[9] N. Grau, Nucl. Phys. A 830, 797C (2009); D. d'Enterria et al., J. Phys. G 34 (2007) 2307.

[10] J. Putschke, this proceedings; J. Jia, this proceedings; M. Ploskon, A. Morsch, Y. Lai, et al, Round Table II: Jet reconstruction in high background environment at RHIC and LHC, Hard Probe 2010

[11] I. Vitev, S. Wicks and B. W. Zhang, JHEP 0811, 093 (2008); I. Vitev, B. W. Zhang and S. Wicks, Eur. Phys. J. C 62, 139 (2009); B. W. Zhang, Nucl. Phys. A830, 259C-262C (2009).

[12] I. Vitev, B. -W. Zhang, Phys. Rev. Lett. 104, 132001 (2010). [arXiv:0910.1090 [hep-ph]].

[13] R. B. Neufeld, I. Vitev, B. -W. Zhang, [arXiv:1006.2389 [hep-ph]].

[14] M. H. Seymour, Nucl. Phys. B 513, 269 (1998).

[15] S. D. Ellis, Z. Kunszt, D. E. Soper, Phys. Rev. Lett. 64, 2121 (1990); Z. Kunszt and D. E. Soper, Phys. Rev. D 46, 192 (1992).

[16] D. G. d'Enterria, |nucl-ex/0302016].

[17] B. I. Abelev et al. [STAR Collaboration], Phys. Rev. Lett. 97, 252001 (2006).

[18] D. E. Acosta et al. [CDF Collaboration], Phys. Rev. D 71, 112002 (2005).

[19] I. Vitev, B. W. Zhang, Phys. Lett. B 669, 337 (2008); R. Sharma, I. Vitev, B. Zhang, Phys. Rev. C 80, 054902 (2009).

[20] R. B. Neufeld, I. Vitev, B. W. Zhang, |arXiv:1010.3708 [hep-ph]].

[21] J. M. Campbell, J. W. Huston and W. J. Stirling, Rept. Prog. Phys. 70, 89 (2007); S. D. Ellis et al. Prog. Part. Nucl. Phys. 60, 484 (2008).

[22] F. I. Olness and D. E. Soper, arXiv:0907.5052 [hep-ph].

[23] M. Dasgupta, L. Magnea, G. Salam, JHEP 0802, 055 (2008).

[24] R. B. Neufeld, this proceedings.

[25] J. M. Campbell and R. K. Ellis, Phys. Rev. D 65, 113007 (2002).

[26] V. M. Abazov et al. [D0 Collaboration], Phys. Lett. B 669, 278 (2008).

[27] H. Li, B. W. Zhang, I. Vitev, and E. Wang, in preparation.

[28] G. P. Salam, Eur. Phys. J. C67, 637-686 (2010).

[29] T. Sjostrand, et al, Comput. Phys. Commun. 178, 852-867 (2008). 